\title{
Effects of hydrogen peroxide treatment on the germination, vigour and health of Zinnia elegans seeds
}

\author{
Dorota Szopińska \\ Department of Phytopathology, Seed Science and Technology \\ Poznań University of Life Sciences \\ Szamotulska 28, Baranowo, 62-081 Przeźmierowo, Poland
}

\begin{abstract}
The high infestation rate of zinnia (Zinnia elegans Jacq.) seeds with fungi, especially pathogenic Alternaria zinniae, has frequently resulted in a poor germination capacity associated with a high number of abnormal diseased seedlings. The effect of hydrogen peroxide $\left(\mathrm{H}_{2} \mathrm{O}_{2}\right)$ treatment on the germination, vigour and health of zinnia seeds was investigated. Two samples of zinnia seeds - sample I, characterised with a low germination capacity (39.5\%) and a high level of seed infection with $A$. zinniae (76\%), and sample II, with a high germination capacity $(87 \%)$ and free from this pathogen - were tested. For treatment, seeds were soaked in $3 \%, 6 \%, 9 \%$ and $12 \% \mathrm{H}_{2} \mathrm{O}_{2}$ solutions for 10, 20, 30 and $60 \mathrm{~min}$. Initially, the controls were seeds soaked in distilled water for 10, 20, 30 and $60 \mathrm{~min}$, and then the results of selected treatments were compared with results obtained for untreated seeds and seeds treated with a fungicide (20\% carbendazim and $45 \%$ thiram). The fungi Alternaria spp. and Fusarium spp. were frequently identified on tested seeds. The concentration of hydrogen peroxide affected the quality of zinnia seeds to a higher extent than the treatment time. However, all of the treatments applied, regardless of time and concentration of $\mathrm{H}_{2} \mathrm{O}_{2}$, positively affected seed health, significantly reducing seed infestation by fungi in both samples. Moreover, the lowest level of $A$. zinniae infection was observed if seeds were soaked in $6 \%$ and $9 \% \mathrm{H}_{2} \mathrm{O}_{2}$ solutions for 60 and $20 \mathrm{~min}$, respectively. Higher concentrations of $\mathrm{H}_{2} \mathrm{O}_{2}(9 \%$ and $12 \%)$ negatively influenced seed germination and vigour, especially in sample II. These findings suggested that the treatment of zinnia seeds with hydrogen peroxide can effectively improve germination mostly in samples heavily infected with pathogens, which means that it should be preceded by seed health evaluation.
\end{abstract}

Key words: $\mathrm{H}_{2} \mathrm{O}_{2}$, seed infestation with fungi, seed quality, zinnia

\section{INTRODUCTION}

Zinnia (Zinnia elegans Jacq.) is a popular annual ornamental plant. However, it is frequently infected with pathogenic fungi. It was found that the fungi Alternaria zinniae, Fusarium culmorum, F. solani, F. oxysporum and Sclerotinia sclerotiorum were in most cases responsible for damage in zinnia plants in the field (Lacicowa et al. 1979). On the other hand, Alternaria alternata, A. zinniae, Botrytis cinerea, Cladosporium spp. Fusarium spp. and Penicillium spp. were frequently detected on zinnia seeds (Łacicowa et al. 1991, Szopińska et al. 2012). Among them, A. zinniae is considered the most important fungal seed-borne pathogen of this plant. The fungus causes spotting of petals, foliage and stems, as well as rotting of roots (Dimock and 
Osborn 1943, Richardson 1990, Łacicowa et al. 1991, Wu and Yang 1992). High seed infestation with fungi, especially Alternaria spp. and Fusarium spp., results in poor germination of zinnia seeds (Szopińska and Tylkowska 2009, Szopińska and Wojtaszek 2011). Chemical compounds have been commonly used to control the health of the seeds. However, there is a worldwide need for a more organic production, including abandoning standard chemical crop protectants (Groot et al. 2004). Hydrogen peroxide $\left(\mathrm{H}_{2} \mathrm{O}_{2}\right)$, characterised by its highly oxidative reactivity, is generated naturally in plant cells under stressful conditions such as chilling, drought, wounding, pathogen intrusion, etc. (Ogawa and Iwabuchi 2001). The antimicrobial properties of $\mathrm{H}_{2} \mathrm{O}_{2}$ have been commonly used in medicine and the food industry (Lehrer 1969, Thomas 1979, Sapers and Sites 2003). Some experiments have also been performed to determine the antimicrobial properties of hydrogen peroxide against plant pathogens (Peng and Kuc 1992, Smilanick et al. 1994, Geetha and Shetty 2002). However, there are few studies on the control of seed-borne pathogens by the treatment of seeds with $\mathrm{H}_{2} \mathrm{O}_{2}$ before sowing (Smilanick et al. 1994, Neumann et al. 1997, Rosada 2012, Słupinska 2012). Moreover, hydrogen peroxide has been reported to improve the germination of zinnia seeds (Ogawa and Iwabuchi 2001), as well as barley (Hordeum vulgare) and wheat (Triticum aestivum) (Smilanick et al. 1994), eastern gamagrass (Tripsacum dactyloides) (Klein et al. 2008), rice (Oryza sativa) (Naredo et al. 1998, Sasaki et al. 2005), maize (Zea mays) (Wahid et al. 2008), watermelon (Citrullus lanatus) (Duval and NeSmith 2000, Jaskani et al. 2006), camphor tree (Cinnamomum camphora) (Chien and Lin 1994), and muscadine (Vitis rotundifolia) (Conner 2008). Ogawa and Iwabuchi (2001) observed that the oxidation of germination inhibitors present in the pericarp by $\mathrm{H}_{2} \mathrm{O}_{2}$ promoted the germination of zinnia seeds. The authors incubated the achenes and seeds without pericarp on a blotter soaked with $\mathrm{H}_{2} \mathrm{O}_{2}$ at a concentration ranging from 1 to $50 \mathrm{mM}$. They found that the concentration $\mathrm{H}_{2} \mathrm{O}_{2}$ giving the maximum promotional effect varied depending on the time following the imbibition of the seeds, which suggests that $\mathrm{H}_{2} \mathrm{O}_{2}$ influences more than two steps of germination. On the other hand, the direct physical scarifying effect of this compound has been also suggested by Klein et al. (2008). Conner (2008) observed that the pre-treatment of muscadine seeds before stratification with hydrogen peroxide and gibberellic acid effectively promoted germination.
Klein et al. (2008) found that treatment with $15 \%$ $\mathrm{H}_{2} \mathrm{O}_{2}$ for $18 \mathrm{~h}$ can substitute six-week stratification at $4^{\circ} \mathrm{C}$ in satisfying the dormancy of eastern gamagrass. Duval and NeSmith (2000) suggested that the germination barriers of watermelon are seed coat related, and that seed coat alteration and $\mathrm{H}_{2} \mathrm{O}_{2}$ can overcome these barriers. Narimanov (2000) observed that a short period of soaking seeds of barley, maize, haricot, melon, vegetable marrow, garden radish, and carrot in $6 \times 10^{-2}$ to $6 \times 10^{-5} \mathrm{M}$ $\mathrm{H}_{2} \mathrm{O}_{2}$ solution enhanced germination, promoted the early appearance of sprouts, and accelerated the development of plants.

Ogawa and Iwabuchi (2011) unravelled how treatment with hydrogen peroxide promotes the germination of zinnia seeds. However, the researchers only studied the frequency of germination, defined as the time when the length of the elongating and extruding part exceeds that of the seed or when the cotyledons opened. These observations were carried only in the initial stage of germination and did not explain how treatment with $\mathrm{H}_{2} \mathrm{O}_{2}$ may affect germination capacity, and other parameters evaluated during a standard germination test, as well as the vigour and health of zinnia seeds.

The present experiment was undertaken to investigate the effect of hydrogen peroxide on the quality of zinnia seeds, with special attention to their germination, vigour and infestation with fungi.

\section{MATERIAL AND METHODS}

Two samples of zinnia seeds: sample I - cultivar Jowita and sample II - cultivar Kirke, produced by the TORSEED S.A. seed company, were used in the experiment. Hydrogen peroxide ( $30 \%$ solution) was obtained from Sigma-Aldrich Co. The fungicide, Sarfun T 65 DS (20\% carbendazim and $45 \%$ thiram), produced by Organika-Sarzyna S.A., was used as an alternative chemical control.

Seeds of each sample were treated with $3 \%$, $6 \%, 9 \%$ and $12 \%$ aqueous solutions of hydrogen peroxide for 10, 20, 30 and $60 \mathrm{~min}$. After the treatment, the seeds were washed under running tap water for $2 \mathrm{~min}$ and then rinsed twice with distilled water. Germination, vigour and health tests were performed for each combination. Initially, the controls were untreated seeds soaked for 10, 20, 30 and $60 \mathrm{~min}$ in distilled water and the results of the germination, vigour and health tests were compared by means of a two-factor variance analysis followed by the Duncan multiple range test. Then, when 
optimum treatment conditions were determined (taking into account the concentration of $\mathrm{H}_{2} \mathrm{O}_{2}$ and treatment time), their efficacy was evaluated in comparison with the results obtained for untreated seeds and seeds treated with fungicide at a dose of $5 \mathrm{~g} \mathrm{~kg}^{-1}$ of seeds, using one-way variance analysis followed by the Duncan multiple range test.

Germination was evaluated using ISTA rules (ISTA 2012). Four hundred seeds from each combination (eight replicates of 50 seeds) were placed on blotters soaked with distilled water in $9 \mathrm{~cm}$ diameter Petri dishes (25 seeds per dish), and incubated at $20^{\circ} \mathrm{C}$ in darkness. The numbers of normal (germination capacity) and abnormal (deformed and diseased) seedlings were determined after four and 10 days of incubation. Additionally, the total seed germination $\left(\mathrm{G}_{\max }\right)$ was calculated during seed vigour evaluation, on the basis of the number of seeds with visible radicles counted daily.

A vigour test was performed on 400 seeds from each combination (eight replicates of 50 seeds) according to the procedure and using the same conditions as described for the germination test. However, germinating seeds with a visible radicle were counted daily for 10 days and removed from the plates. SeedCalculator 1.2 software (Jalink and Van der Schoor 1999) was used for analysing vigour parameters, i.e. $\mathrm{T}_{1}-$ time to $1 \%$ of germinating seeds $\left(\mathrm{G}_{\max }\right)$ and MGT - mean germination time.

The deep freeze blotter test was performed to determine the health of the seeds. Two hundred seeds from each combination (four replicates of
50 seeds) were placed on blotters soaked with distilled water in $9 \mathrm{~cm}$ diameter Petri dishes (10 seeds per plate). The seeds were kept at $20^{\circ} \mathrm{C}$ in darkness for $24 \mathrm{~h}$, then frozen at $-20^{\circ} \mathrm{C}$ for $24 \mathrm{~h}$ and then incubated at $20^{\circ} \mathrm{C}$ for eight days under an alternating cycle of NUV light and darkness (12 $\mathrm{h}$ of light and $12 \mathrm{~h}$ of darkness). After incubation the fungi appearing on the seeds were identified on the basis of their growth and sporulation using a stereomicroscope, and compound microscope if necessary (Machado et al. 2002, Mathur and Kongsdal 2003). Additionally, the percentage of seeds free from fungi was calculated.

\section{RESULTS}

The effects of hydrogen peroxide treatment, considering the concentration of $\mathrm{H}_{2} \mathrm{O}_{2}$ and treatment time, on the germination, vigour and health of zinnia seeds, were determined initially in comparison to seeds soaked in distilled water (Tabs 1-9).

Hydrogen peroxide treatment, regardless of concentration, did not significantly influence the total germination $\left(\mathrm{G}_{\max }\right)$ of sample II seeds (Tab. 1). In contrast, a decrease of $G_{\max }$ was observed in sample I when seeds were treated in $6 \%, 9 \%$ and $12 \%$ hydrogen peroxide solutions. Sample I was characterised by a low germination capacity and the treatment of seeds with hydrogen peroxide at a concentration of $6 \%, 9 \%$ and $12 \%$ decreased this parameter (Tab. 2). Hydrogen peroxide at the highest concentration also negatively influenced

Table 1. Effects of hydrogen peroxide treatment on the total germination of zinnia seeds $\left(\mathrm{G}_{\max }\right)$

\begin{tabular}{|c|c|c|c|c|c|c|}
\hline \multirow{2}{*}{ Sample } & \multirow{2}{*}{ Treatment } & \multicolumn{4}{|c|}{ Treatment time (min) } & \multirow[b]{2}{*}{ Mean } \\
\hline & & 10 & 20 & 30 & 60 & \\
\hline \multirow[t]{6}{*}{ I } & Water* & 83.3 a-c** & $84.0 \mathrm{c}$ & 82.3 a-c & 82.0 a-c & $82.9 \mathrm{c}$ \\
\hline & $\mathrm{H}_{2} \mathrm{O}_{2} 3 \%$ & 84.0 bc & $84.8 \mathrm{c}$ & 82.0 a-c & 79.0 a-c & $82.5 \mathrm{bc}$ \\
\hline & $\mathrm{H}_{2} \mathrm{O}_{2} 6 \%$ & $77.0 \mathrm{ab}$ & 78.3 a-c & $81.3 \mathrm{a}-\mathrm{c}$ & $76.3 \mathrm{a}$ & $78.2 \mathrm{a}$ \\
\hline & $\mathrm{H}_{2} \mathrm{O}_{2} 9 \%$ & 82.5 a-c & 79.8 a-c & 79.5 a-c & $76.3 \mathrm{a}$ & $79.5 \mathrm{ab}$ \\
\hline & $\mathrm{H}_{2} \mathrm{O}_{2} 12 \%$ & 80.5 a-c & 80.0 a-c & 79.0 a-c & 79.3 a-c & $79.7 \mathrm{ab}$ \\
\hline & Mean & $81.5 \mathrm{~b}$ & $81.4 \mathrm{~b}$ & $80.8 \mathrm{ab}$ & 78.6 a & \\
\hline \multirow[t]{6}{*}{ II } & Water & $95.5 \mathrm{ab}$ & $94.0 \mathrm{ab}$ & $96.3 \mathrm{~b}$ & $93.0 \mathrm{ab}$ & $94.7 \mathrm{a}$ \\
\hline & $\mathrm{H}_{2} \mathrm{O}_{2} 3 \%$ & $93.5 \mathrm{ab}$ & $92.0 \mathrm{a}$ & $96.0 \mathrm{ab}$ & $95.3 \mathrm{ab}$ & $94.2 \mathrm{a}$ \\
\hline & $\mathrm{H}_{2} \mathrm{O}_{2} 6 \%$ & $93.0 \mathrm{ab}$ & $94.3 \mathrm{ab}$ & $93.3 \mathrm{ab}$ & $92.3 \mathrm{a}$ & $93.2 \mathrm{a}$ \\
\hline & $\mathrm{H}_{2} \mathrm{O}_{2} 9 \%$ & $92.8 \mathrm{ab}$ & $92.3 \mathrm{a}$ & $93.5 \mathrm{ab}$ & $94.8 \mathrm{ab}$ & $93.4 \mathrm{a}$ \\
\hline & $\mathrm{H}_{2} \mathrm{O}_{2} 12 \%$ & $92.5 \mathrm{a}$ & $92.8 \mathrm{ab}$ & $94.5 \mathrm{ab}$ & $91.8 \mathrm{a}$ & 92.9 a \\
\hline & Mean & $93.5 \mathrm{ab}$ & $93.1 \mathrm{a}$ & $94.7 \mathrm{~b}$ & $93.4 \mathrm{ab}$ & \\
\hline
\end{tabular}

*Water - seeds soaked in distilled water; $\mathrm{H}_{2} \mathrm{O}_{2} 3 \%, \mathrm{H}_{2} \mathrm{O}_{2} 6 \%, \mathrm{H}_{2} \mathrm{O}_{2} 9 \%, \mathrm{H}_{2} \mathrm{O}_{2} 12 \%$ - seeds soaked in 3\%, 6\%, 9\% and $12 \%$ hydrogen peroxide solutions, respectively

**Means followed by the same letters, for each sample separately, are not significantly different according to the Duncan multiple range test $(\alpha=0.05)$ 
Table 2. Effects of hydrogen peroxide treatment on germination capacity of zinnia seeds (\%)

\begin{tabular}{|c|c|c|c|c|c|c|}
\hline \multirow{2}{*}{ Sample } & \multirow{2}{*}{ Treatment } & \multicolumn{4}{|c|}{ Treatment time $(\mathrm{min})$} & \multirow[b]{2}{*}{ Mean } \\
\hline & & 10 & 20 & 30 & 60 & \\
\hline \multirow[t]{6}{*}{ I } & Water & 48.3 c-e & 57.8 ef & 57.0 ef & $62.5 \mathrm{f}$ & $56.4 \mathrm{~b}$ \\
\hline & $\mathrm{H}_{2} \mathrm{O}_{2} 3 \%$ & $47.8 \quad \mathrm{c}-\mathrm{e}$ & $50.3 \mathrm{c}-\mathrm{e}$ & $51.8 \mathrm{c}-\mathrm{f}$ & $56.5 \mathrm{~d}-\mathrm{f}$ & $51.6 \mathrm{~b}$ \\
\hline & $\mathrm{H}_{2} \mathrm{O}_{2} 6 \%$ & $43.3 \mathrm{bc}$ & $42.8 \mathrm{bc}$ & $45.0 \mathrm{~cd}$ & $40.3 \mathrm{a}-\mathrm{c}$ & $42.9 \mathrm{a}$ \\
\hline & $\mathrm{H}_{2} \mathrm{O}_{2} 9 \%$ & $47.8 \mathrm{c}-\mathrm{e}$ & $46.8 \mathrm{c}-\mathrm{e}$ & $42.3 \mathrm{a}-\mathrm{c}$ & $32.0 \mathrm{a}$ & $42.2 \mathrm{a}$ \\
\hline & $\mathrm{H}_{2} \mathrm{O}_{2} 12 \%$ & $52.3 \mathrm{c}-\mathrm{f}$ & $48.0 \quad \mathrm{c}-\mathrm{e}$ & $46.3 \mathrm{c}-\mathrm{e}$ & $33.3 \mathrm{ab}$ & $45.0 \mathrm{a}$ \\
\hline & Mean & $47.9 \mathrm{a}$ & $49.1 \mathrm{a}$ & $48.5 \mathrm{a}$ & $44.9 \mathrm{a}$ & \\
\hline \multirow[t]{6}{*}{ II } & Water & 82.3 e-h & $60.0 \mathrm{~b}$ & $68.3 \mathrm{~b}-\mathrm{d}$ & 72.5 b-g & $70.8 \mathrm{~b}$ \\
\hline & $\mathrm{H}_{2} \mathrm{O}_{2} 3 \%$ & $75.0 \mathrm{c}-\mathrm{h}$ & $75.5 \mathrm{c}-\mathrm{h}$ & $77.8 \mathrm{c}-\mathrm{h}$ & $84.5 \mathrm{gh}$ & $78.2 \mathrm{c}$ \\
\hline & $\mathrm{H}_{2} \mathrm{O}_{2} 6 \%$ & $78.0 \mathrm{c}-\mathrm{h}$ & $82.3 \mathrm{f}-\mathrm{h}$ & $87.0 \mathrm{~h}$ & 80.0 d-h & $81.8 \mathrm{c}$ \\
\hline & $\mathrm{H}_{2} \mathrm{O}_{2} 9 \%$ & $67.5 \mathrm{~b}-\mathrm{d}$ & 70.5 b-e & 73.0 b-g & 71.0 b-f & $70.5 \mathrm{~b}$ \\
\hline & $\mathrm{H}_{2} \mathrm{O}_{2} 12 \%$ & $60.0 \mathrm{~b}$ & $42.5 \mathrm{a}$ & $66.3 \mathrm{bc}$ & $43.3 \mathrm{a}$ & $53.0 \mathrm{a}$ \\
\hline & Mean & $72.6 \mathrm{~b}$ & $66.2 \mathrm{a}$ & $74.5 \mathrm{~b}$ & $70.3 \mathrm{ab}$ & \\
\hline
\end{tabular}

Explanations: see Table 1

Table 3. Effects of hydrogen peroxide treatment on percentage of abnormal deformed seedlings

\begin{tabular}{|c|c|c|c|c|c|c|}
\hline \multirow{2}{*}{ Sample } & \multirow{2}{*}{ Treatment } & \multicolumn{4}{|c|}{ Treatment time (min) } & \multirow[b]{2}{*}{ Mean } \\
\hline & & 10 & 20 & 30 & 60 & \\
\hline \multirow[t]{6}{*}{ I } & Water & $0.3 \mathrm{ab}$ & $0 \mathrm{a}$ & $0 \mathrm{a}$ & $0 \mathrm{a}$ & $0.1 \mathrm{a}$ \\
\hline & $\mathrm{H}_{2} \mathrm{O}_{2} 3 \%$ & $1.3 \mathrm{a}-\mathrm{c}$ & $1.3 \mathrm{a}-\mathrm{c}$ & $2.5 \mathrm{c}$ & $1.5 \mathrm{a}-\mathrm{c}$ & $1.7 \mathrm{~b}$ \\
\hline & $\mathrm{H}_{2} \mathrm{O}_{2} 6 \%$ & $0.5 \mathrm{ab}$ & $1.0 \mathrm{a}-\mathrm{c}$ & 0.8 a-c & $1.3 \mathrm{a}-\mathrm{c}$ & $0.9 \mathrm{~b}$ \\
\hline & $\mathrm{H}_{2} \mathrm{O}_{2} 9 \%$ & $1.0 \mathrm{a}-\mathrm{c}$ & $1.0 \mathrm{a}-\mathrm{c}$ & $1.3 \mathrm{a}-\mathrm{c}$ & $1.0 \mathrm{a}-\mathrm{c}$ & $1.1 \mathrm{~b}$ \\
\hline & $\mathrm{H}_{2} \mathrm{O}_{2} 12 \%$ & $1.5 \mathrm{a}-\mathrm{c}$ & $2.0 \mathrm{bc}$ & $1.3 \mathrm{a}-\mathrm{c}$ & $1.0 \mathrm{ab}$ & $1.5 \mathrm{~b}$ \\
\hline & Mean & 0.9 a & $1.1 \mathrm{a}$ & $1.2 \mathrm{a}$ & $1.0 \mathrm{a}$ & \\
\hline \multirow[t]{6}{*}{ II } & Water & $0 \mathrm{a}$ & $0 \mathrm{a}$ & $0 \mathrm{a}$ & $0 \mathrm{a}$ & $0 \mathrm{a}$ \\
\hline & $\mathrm{H}_{2} \mathrm{O}_{2} 3 \%$ & $0.3 \mathrm{ab}$ & $4.3 \mathrm{c}-\mathrm{f}$ & $2.5 \mathrm{~cd}$ & $3.0 \mathrm{c}-\mathrm{e}$ & $2.5 \mathrm{~b}$ \\
\hline & $\mathrm{H}_{2} \mathrm{O}_{2} 6 \%$ & $1.0 \mathrm{a}-\mathrm{c}$ & $2.3 \mathrm{~b}-\mathrm{d}$ & $2.3 \mathrm{~b}-\mathrm{d}$ & $5.3 \mathrm{~d}-\mathrm{f}$ & $2.7 \mathrm{~b}$ \\
\hline & $\mathrm{H}_{2} \mathrm{O}_{2} 9 \%$ & 8.5 e-g & $5.8 \mathrm{~d}-\mathrm{f}$ & $5.8 \mathrm{~d}-\mathrm{f}$ & $8.5 \mathrm{fg}$ & $7.2 \mathrm{c}$ \\
\hline & $\mathrm{H}_{2} \mathrm{O}_{2} 12 \%$ & $24.3 \mathrm{i}$ & $31.0 \mathrm{i}$ & $11.3 \mathrm{gh}$ & $16.5 \mathrm{~h}$ & 20.8 \\
\hline & Mean & $6.8 \mathrm{ab}$ & $8.7 \mathrm{c}$ & $4.4 \mathrm{a}$ & $6.7 \mathrm{bc}$ & \\
\hline
\end{tabular}

Explanations: see Table 1

the germination of sample II seeds, while at concentrations of $3 \%$ and $6 \%$ it significantly improved this parameter. Generally, soaking seeds in $\mathrm{H}_{2} \mathrm{O}_{2}$ solutions resulted in an increase in the percentage of abnormal deformed seedlings in both samples (Tab. 3). Moreover, an increase in the percentage of abnormal diseased seedlings was observed when seeds of sample I were treated with $6 \%$ and $9 \% \mathrm{H}_{2} \mathrm{O}_{2}$ (Tab. 4). However, the percentage of these seedlings decreased when seeds of sample II were treated with $3 \%, 6 \%$ and $9 \% \mathrm{H}_{2} \mathrm{O}_{2}$. Treatment time affected the germination parameters to a smaller extent than $\mathrm{H}_{2} \mathrm{O}_{2}$ concentration, and was observed mostly in sample II, where the lowest value of germination capacity and the highest percentage of abnormal deformed seedlings were observed after the 20 min treatment.

The prolongation of time needed to $1 \%$ of $\mathrm{G}_{\max }$ as well as prolongation of mean germination time was observed when seeds of sample I were treated in $3 \%, 9 \%$ and $12 \%$ solutions of $\mathrm{H}_{2} \mathrm{O}_{2}$ (Tabs 5 and 6). Hydrogen peroxide treatment, regardless of concentration, also negatively influenced these parameters in sample II. However, the highest values of $\mathrm{T}_{1}$ and MGT were observed if the seeds were soaked in $9 \%$ and $12 \%$ solutions of $\mathrm{H}_{2} \mathrm{O}_{2}$. Generally, the prolongation of treatment time over $20 \mathrm{~min}$ in the case of $\mathrm{T}_{1}$, and over $10 \mathrm{~min}$ in the case of mean germination time, negatively affected seed vigour in both samples. 
Table 4. Effects of hydrogen peroxide treatment on percentage of abnormal diseased seedlings

\begin{tabular}{|c|c|c|c|c|c|c|}
\hline \multirow{2}{*}{ Sample } & \multirow{2}{*}{ Treatment } & \multicolumn{4}{|c|}{ Treatment time $(\mathrm{min})$} & \multirow[b]{2}{*}{ Mean } \\
\hline & & 10 & 20 & 30 & 60 & \\
\hline \multirow[t]{6}{*}{ I } & Water & $33.3 \mathrm{~d}-\mathrm{f}$ & $23.0 \mathrm{a}-\mathrm{c}$ & $21.0 \mathrm{ab}$ & $19.5 \mathrm{a}$ & $24.2 \mathrm{a}$ \\
\hline & $\mathrm{H}_{2} \mathrm{O}_{2} 3 \%$ & 30.8 b-f & $30.0 \mathrm{~b}-\mathrm{f}$ & 25.8 a-d & $19.0 \mathrm{a}$ & $26.4 \mathrm{a}$ \\
\hline & $\mathrm{H}_{2} \mathrm{O}_{2} 6 \%$ & $38.8 \mathrm{f}$ & 37.0 ef & 26.8 a-e & $34.3 \mathrm{~d}-\mathrm{f}$ & $34.2 \mathrm{c}$ \\
\hline & $\mathrm{H}_{2} \mathrm{O}_{2} 9 \%$ & $31.3 \mathrm{c}-\mathrm{f}$ & $33.3 \mathrm{~d}-\mathrm{f}$ & $30.0 \mathrm{~b}-\mathrm{f}$ & $33.5 \mathrm{~d}-\mathrm{f}$ & $32.0 \mathrm{bc}$ \\
\hline & $\mathrm{H}_{2} \mathrm{O}_{2} 12 \%$ & $25.3 \mathrm{a}-\mathrm{d}$ & $25.8 \mathrm{a}-\mathrm{d}$ & 27.3 a-e & $33.8 \mathrm{~d}-\mathrm{f}$ & $28.1 \mathrm{ab}$ \\
\hline & Mean & $31.9 \mathrm{~b}$ & $29.8 \mathrm{ab}$ & $26.2 \mathrm{a}$ & $28.0 \mathrm{ab}$ & \\
\hline \multirow[t]{6}{*}{ II } & Water & $9.0 \mathrm{a}-\mathrm{c}$ & $31.8 \mathrm{f}$ & $23.5 \mathrm{~d}-\mathrm{f}$ & $19.8 \mathrm{c}-\mathrm{f}$ & $21.0 \mathrm{c}$ \\
\hline & $\mathrm{H}_{2} \mathrm{O}_{2} 3 \%$ & $14.8 \mathrm{a}-\mathrm{d}$ & $15.0 \mathrm{a}-\mathrm{d}$ & $12.5 \mathrm{a}-\mathrm{d}$ & $5.8 \mathrm{ab}$ & $12.0 \mathrm{ab}$ \\
\hline & $\mathrm{H}_{2} \mathrm{O}_{2} 6 \%$ & $13.5 \mathrm{a}-\mathrm{d}$ & $8.3 \mathrm{ab}$ & $4.5 \mathrm{a}$ & $8.5 \mathrm{ab}$ & $8.7 \mathrm{a}$ \\
\hline & $\mathrm{H}_{2} \mathrm{O}_{2} 9 \%$ & $17.0 \mathrm{~b}-\mathrm{e}$ & $16.0 \mathrm{~b}-\mathrm{e}$ & 10.8 a-d & 9.0 a-c & $13.2 \mathrm{~b}$ \\
\hline & $\mathrm{H}_{2} \mathrm{O}_{2} 12 \%$ & $8.0 \mathrm{a}-\mathrm{c}$ & $16.0 \mathrm{a}-\mathrm{d}$ & $12.0 \mathrm{a}-\mathrm{d}$ & 30.0 ef & $16.5 \mathrm{bc}$ \\
\hline & Mean & $12.5 \mathrm{a}$ & $17.4 \mathrm{a}$ & $12.7 \mathrm{a}$ & $14.6 \mathrm{a}$ & \\
\hline
\end{tabular}

Explanations: see Table 1

Table 5. Effects of hydrogen peroxide treatment on time needed to reach $1 \%$ of total germinating zinnia seeds (days)

\begin{tabular}{|c|c|c|c|c|c|c|}
\hline \multirow{2}{*}{ Sample } & \multirow{2}{*}{ Treatment } & \multicolumn{4}{|c|}{ Treatment time (min) } & \multirow[b]{2}{*}{ Mean } \\
\hline & & 10 & 20 & 30 & 60 & \\
\hline \multirow[t]{6}{*}{ I } & Water & $0.26 \mathrm{a}$ & $0.24 \mathrm{a}$ & $0.27 \mathrm{a}$ & $0.27 \mathrm{a}$ & $0.30 \mathrm{a}$ \\
\hline & $\mathrm{H}_{2} \mathrm{O}_{2} 3 \%$ & $0.44 \mathrm{a}$ & $0.77 \mathrm{~b}-\mathrm{d}$ & 0.44 a-c & $0.60 \mathrm{~cd}$ & $0.50 \mathrm{c}$ \\
\hline & $\mathrm{H}_{2} \mathrm{O}_{2} 6 \%$ & $0.24 \mathrm{a}$ & $0.27 \mathrm{a}$ & $0.26 \mathrm{a}$ & $0.38 \mathrm{ab}$ & $0.34 \mathrm{ab}$ \\
\hline & $\mathrm{H}_{2} \mathrm{O}_{2} 9 \%$ & $0.22 \mathrm{a}$ & $0.33 \mathrm{a}$ & $0.35 \mathrm{a}$ & $0.64 \mathrm{~cd}$ & $0.42 \mathrm{bc}$ \\
\hline & $\mathrm{H}_{2} \mathrm{O}_{2} 12 \%$ & $0.28 \mathrm{a}$ & $0.35 \mathrm{a}$ & $0.80 \mathrm{~d}$ & 0.46 a-c & $0.49 \mathrm{c}$ \\
\hline & Mean & $0.30 \mathrm{a}$ & $0.39 \mathrm{ab}$ & $0.46 \mathrm{bc}$ & $0.49 \mathrm{c}$ & \\
\hline \multirow[t]{6}{*}{ II } & Water & 0.29 a-c & 0.56 a-c & $0.60 \mathrm{ab}$ & $0.20 \mathrm{a}$ & $0.36 \mathrm{a}$ \\
\hline & $\mathrm{H}_{2} \mathrm{O}_{2} 3 \%$ & $0.34 \mathrm{a}-\mathrm{c}$ & $0.74 \mathrm{~d}-\mathrm{h}$ & 0.86 g-i & $0.70 \mathrm{~d}-\mathrm{g}$ & $0.68 \mathrm{~b}$ \\
\hline & $\mathrm{H}_{2} \mathrm{O}_{2} 6 \%$ & $0.78 \mathrm{~d}-\mathrm{f}$ & 0.48 b-d & $0.59 \mathrm{c}-\mathrm{e}$ & $0.82 \mathrm{f}-\mathrm{h}$ & $0.65 \mathrm{~b}$ \\
\hline & $\mathrm{H}_{2} \mathrm{O}_{2} 9 \%$ & $0.66 \mathrm{~d}-\mathrm{g}$ & $0.80 \mathrm{~g}-\mathrm{i}$ & $0.78 \mathrm{e}-\mathrm{h}$ & 0.85 hi & $0.84 \mathrm{c}$ \\
\hline & $\mathrm{H}_{2} \mathrm{O}_{2} 12 \%$ & $0.89 \mathrm{~g}-\mathrm{i}$ & $0.83 \mathrm{~g}-\mathrm{i}$ & $0.97 \mathrm{j}$ & $1.00 \mathrm{ij}$ & $1.02 \mathrm{~d}$ \\
\hline & Mean & $0.61 \mathrm{a}$ & $0.69 \mathrm{ab}$ & $0.76 \mathrm{~b}$ & $0.77 \mathrm{~b}$ & \\
\hline
\end{tabular}

Explanations: see Table 1

Treatment with hydrogen peroxide, regardless of concentration, positively affected the health of the seeds, significantly increasing the percentage of seeds free from fungi in both samples (Tab. 7). However, the highest percentage of these seeds was observed when the seeds of both samples were soaked in a $12 \%$ solution of $\mathrm{H}_{2} \mathrm{O}_{2}$. The effect of treatment time was less significant and observed only in sample I, where the prolongation of the treatment over $10 \mathrm{~min}$ increased the percentage of seeds free from fungi. Alternaria alternata (Fr.) Keissler and Fusarium spp. were frequently identified on the seeds of both samples (Tabs 8 and 9). Additionally, Alternaria zinniae Pape was often detected on the seeds of sample I. Soaking seeds of both samples in hydrogen peroxide solutions, in all applied concentrations, significantly reduced seed infestation with Alternaria spp. and Fusarium spp., whereas the lowest percentage of seeds infested with these fungi was observed after the treatment with $12 \% \mathrm{H}_{2} \mathrm{O}_{2}$. The prolongation of treatment time resulted in a decrease of seed infestation with $A$. alternata and $A$. zinniae in sample $\mathrm{I}$, whereas it had no influence on the percentage of seeds infested with A. alternata spp. in sample II and Fusarium spp. in both samples.

Two optimal combinations of seed treatment were selected, considering the influence of $\mathrm{H}_{2} \mathrm{O}_{2}$ concentration and treatment time on the germination, vigour and health of zinnia seeds in both samples, i.e. treating seeds with $3 \% \mathrm{H}_{2} \mathrm{O}_{2}$ for 10 and $20 \mathrm{~min}$. The results of these treatments were 
Table 6. Effects of hydrogen peroxide treatment on mean germination time of zinnia seeds (days)

\begin{tabular}{|c|c|c|c|c|c|c|}
\hline \multirow{2}{*}{ Sample } & \multirow{2}{*}{ Treatment } & \multicolumn{4}{|c|}{ Treatment time $(\mathrm{min})$} & \multirow[b]{2}{*}{ Mean } \\
\hline & & 10 & 20 & 30 & 60 & \\
\hline \multirow[t]{6}{*}{ I } & Water & $1.21 \mathrm{a}$ & $1.34 \mathrm{a}-\mathrm{c}$ & $1.20 \mathrm{a}$ & $1.26 \mathrm{ab}$ & $1.25 \mathrm{a}$ \\
\hline & $\mathrm{H}_{2} \mathrm{O}_{2} 3 \%$ & $1.31 \mathrm{a}-\mathrm{c}$ & $1.48 \mathrm{~cd}$ & $1.43 \mathrm{~b}-\mathrm{d}$ & $1.74 \mathrm{e}$ & $1.48 \mathrm{~b}$ \\
\hline & $\mathrm{H}_{2} \mathrm{O}_{2} 6 \%$ & $1.20 \mathrm{a}$ & $1.37 \mathrm{a}-\mathrm{c}$ & $1.34 \mathrm{a}-\mathrm{c}$ & $1.33 \mathrm{a}-\mathrm{c}$ & $1.31 \mathrm{a}$ \\
\hline & $\mathrm{H}_{2} \mathrm{O}_{2} 9 \%$ & $1.33 \mathrm{a}-\mathrm{c}$ & $1.47 \mathrm{~cd}$ & $1.47 \mathrm{~cd}$ & $1.72 \mathrm{e}$ & $1.50 \mathrm{~b}$ \\
\hline & $\mathrm{H}_{2} \mathrm{O}_{2} 12 \%$ & $1.55 \mathrm{c}$ & $1.75 \mathrm{e}$ & $2.12 \mathrm{f}$ & $1.97 \mathrm{f}$ & $1.84 \mathrm{c}$ \\
\hline & Mean & $1.32 \mathrm{a}$ & $1.48 \mathrm{~b}$ & $1.51 \mathrm{~b}$ & $1.61 \mathrm{c}$ & \\
\hline \multirow[t]{6}{*}{ II } & Water & $1.37 \mathrm{bc}$ & $1.37 \mathrm{bc}$ & $1.31 \mathrm{ab}$ & $1.20 \mathrm{a}$ & $1.31 \mathrm{a}$ \\
\hline & $\mathrm{H}_{2} \mathrm{O}_{2} 3 \%$ & $1.59 \mathrm{de}$ & $1.56 \mathrm{de}$ & 1.71 ef & $1.52 \mathrm{~cd}$ & $1.59 \mathrm{~b}$ \\
\hline & $\mathrm{H}_{2} \mathrm{O}_{2} 6 \%$ & $1.38 \mathrm{bc}$ & $1.60 \mathrm{de}$ & $1.51 \mathrm{~cd}$ & 1.72 ef & $1.55 \mathrm{~b}$ \\
\hline & $\mathrm{H}_{2} \mathrm{O}_{2} 9 \%$ & $1.83 \mathrm{fg}$ & $1.99 \mathrm{~g}$ & $1.87 \mathrm{fg}$ & $2.00 \mathrm{~g}$ & $1.92 \mathrm{c}$ \\
\hline & $\mathrm{H}_{2} \mathrm{O}_{2} 12 \%$ & $1.87 \mathrm{fg}$ & $1.98 \mathrm{~g}$ & $2.18 \mathrm{~h}$ & $2.40 \mathrm{i}$ & $2.11 \mathrm{~d}$ \\
\hline & Mean & $1.61 \mathrm{a}$ & $1.70 \mathrm{~b}$ & $1.72 \mathrm{~b}$ & $1.77 \mathrm{~b}$ & \\
\hline
\end{tabular}

Explanations: see Table 1

Table 7. Effects of hydrogen peroxide treatment on the percentage of zinnia seeds free from fungi

\begin{tabular}{|c|c|c|c|c|c|c|}
\hline \multirow{2}{*}{ Sample } & \multirow{2}{*}{ Treatment } & \multicolumn{4}{|c|}{ Treatment time (min) } & \multirow[b]{2}{*}{ Mean } \\
\hline & & 10 & 20 & 30 & 60 & \\
\hline \multirow[t]{6}{*}{ I } & Water & $0 \mathrm{a}$ & $0 \mathrm{a}$ & $0 \mathrm{a}$ & $0 \mathrm{a}$ & $0 \mathrm{a}$ \\
\hline & $\mathrm{H}_{2} \mathrm{O}_{2} 3 \%$ & $10.5 \mathrm{~b}$ & $16.5 \mathrm{~b}-\mathrm{d}$ & $14.5 \mathrm{bc}$ & $19.5 \mathrm{c}-\mathrm{e}$ & $15.3 \mathrm{~b}$ \\
\hline & $\mathrm{H}_{2} \mathrm{O}_{2} 6 \%$ & $14.0 \mathrm{bc}$ & $22.0 \mathrm{c}-\mathrm{f}$ & $22.0 \mathrm{c}-\mathrm{e}$ & $22.5 \mathrm{c}-\mathrm{f}$ & $20.1 \mathrm{c}$ \\
\hline & $\mathrm{H}_{2} \mathrm{O}_{2} 9 \%$ & $17.5 \mathrm{~b}-\mathrm{e}$ & $21.5 \mathrm{c}-\mathrm{e}$ & $22.5 \mathrm{e}-\mathrm{g}$ & $18.5 \mathrm{~b}-\mathrm{e}$ & $20.0 \mathrm{c}$ \\
\hline & $\mathrm{H}_{2} \mathrm{O}_{2} 12 \%$ & $25.0 \mathrm{~d}-\mathrm{f}$ & $32.5 \mathrm{f}-\mathrm{h}$ & $36.0 \mathrm{gh}$ & $41.5 \mathrm{~h}$ & $33.8 \mathrm{c}$ \\
\hline & Mean & $13.4 \mathrm{a}$ & $18.5 \mathrm{~b}$ & $19.0 \mathrm{~b}$ & $20.4 \mathrm{~b}$ & \\
\hline \multirow[t]{6}{*}{ II } & Water & $0.5 \mathrm{a}$ & $1.5 \mathrm{a}$ & $1.0 \mathrm{a}$ & $1.0 \mathrm{a}$ & $1.0 \mathrm{a}$ \\
\hline & $\mathrm{H}_{2} \mathrm{O}_{2} 3 \%$ & 41.0 b-f & $50.5 \mathrm{c}-\mathrm{g}$ & $52.5 \mathrm{~d}-\mathrm{g}$ & $49.5 \mathrm{c}-\mathrm{g}$ & $48.4 \mathrm{c}$ \\
\hline & $\mathrm{H}_{2} \mathrm{O}_{2} 6 \%$ & $46.5 \mathrm{~b}-\mathrm{g}$ & $50.5 \mathrm{c}-\mathrm{g}$ & $29.5 \mathrm{bc}$ & $30.0 \mathrm{~b}$ & $39.1 \mathrm{~b}$ \\
\hline & $\mathrm{H}_{2} \mathrm{O}_{2} 9 \%$ & $37.0 \mathrm{~b}-\mathrm{e}$ & $50.0 \mathrm{c}-\mathrm{g}$ & $37.0 \mathrm{~b}-\mathrm{d}$ & $30.5 \mathrm{bc}$ & $38.6 \mathrm{~b}$ \\
\hline & $\mathrm{H}_{2} \mathrm{O}_{2} 12 \%$ & $57.0 \quad \mathrm{e}-\mathrm{g}$ & $62.5 \mathrm{fg}$ & $61.0 \mathrm{fg}$ & $66.0 \mathrm{~g}$ & $61.6 \mathrm{c}$ \\
\hline & Mean & $36.4 \mathrm{a}$ & $43.0 \mathrm{a}$ & $36.2 \mathrm{a}$ & $35.4 \mathrm{a}$ & \\
\hline
\end{tabular}

Explanations: see Table 1

compared with the results obtained for untreated seeds (control) and seeds treated with fungicide (Tabs 10-12).

The selected treatments did not influence total seed germination in both samples (Tab. 10). Germination capacity increased significantly after treatment with $3 \% \mathrm{H}_{2} \mathrm{O}_{2}$ for $20 \mathrm{~min}$, as well as after fungicide treatment in sample I. However, in sample II the hydrogen peroxide treatment, regardless of time, as well as the fungicide treatment, negatively affected this parameter. The percentage of abnormal deformed seedlings increased only in sample II if seeds were treated with fungicide and soaked for $20 \mathrm{~min}$ in $3 \% \mathrm{H}_{2} \mathrm{O}_{2}$. Hydrogen peroxide, regardless of treatment time, decreased the percentage of diseased abnormal seedlings in sample I; however, in sample II an increase of the percentage of diseased seedlings was observed after these treatments. Fungicide treatment, as opposed to hydrogen peroxide, negatively affected seed vigour in both samples (Tab. 11). On the other hand, soaking the seeds of both samples in $3 \%$ $\mathrm{H}_{2} \mathrm{O}_{2}$ solution for $10 \mathrm{~min}$ significantly limited the time needed to reach $1 \%$ of germinating seeds.

Hydrogen peroxide at a $3 \%$ concentration, regardless of treatment time, positively affected seed health, increasing the percentage of seeds free from fungi and decreasing seed infestation with Alternaria spp. and Fusarium spp. in both samples (Tab. 12). In sample I, the effectiveness of hydrogen peroxide treatment was frequently similar to the effectiveness of fungicide treatment. 
Table 8. Effects of hydrogen peroxide treatment on the occurrence of fungi on sample I seeds (\%)

\begin{tabular}{|c|c|c|c|c|c|c|}
\hline \multirow{2}{*}{ Fungus } & \multirow{2}{*}{ Treatment } & \multicolumn{4}{|c|}{ Treatment time (min) } & \multirow[b]{2}{*}{ Mean } \\
\hline & & 10 & 20 & 30 & 60 & \\
\hline Alternaria & Water & $75.0 \mathrm{e}^{*}$ & $75.5 \mathrm{e}$ & $70.0 \mathrm{e}$ & $72.0 \mathrm{e}$ & $73.1 \mathrm{~d}$ \\
\hline \multirow[t]{5}{*}{ alternata } & $\mathrm{H}_{2} \mathrm{O}_{2} 3 \%$ & $48.0 \mathrm{~d}$ & $36.0 \mathrm{~b}-\mathrm{d}$ & $45.5 \mathrm{~cd}$ & $41.5 \mathrm{~b}-\mathrm{d}$ & $42.8 \mathrm{c}$ \\
\hline & $\mathrm{H}_{2} \mathrm{O}_{2} 6 \%$ & $37.0 \mathrm{~b}-\mathrm{d}$ & $34.0 \mathrm{~b}-\mathrm{d}$ & $32.0 \mathrm{bc}$ & $33.5 \mathrm{bc}$ & $34.1 \mathrm{~b}$ \\
\hline & $\mathrm{H}_{2} \mathrm{O}_{2} 9 \%$ & $41.0 \quad b-d$ & $37.5 \mathrm{~b}-\mathrm{d}$ & $35.0 \mathrm{~b}-\mathrm{d}$ & $31.5 \mathrm{bc}$ & $36.3 \mathrm{~b}$ \\
\hline & $\mathrm{H}_{2} \mathrm{O}_{2} 12 \%$ & $33.5 \mathrm{~b}-\mathrm{d}$ & $32.0 \mathrm{bc}$ & $27.5 \mathrm{ab}$ & $19.5 \mathrm{a}$ & $28.1 \mathrm{a}$ \\
\hline & Mean & $46.9 \mathrm{~b}$ & $43.0 \mathrm{ab}$ & $42.0 \mathrm{ab}$ & $39.6 \mathrm{a}$ & \\
\hline \multirow{6}{*}{$\begin{array}{l}\text { Alternaria } \\
\text { zinniae }\end{array}$} & Water & $81.0 \mathrm{f}$ & $84.0 \mathrm{f}$ & $80.0 \mathrm{f}$ & $77.0 \mathrm{f}$ & $80.5 \mathrm{c}$ \\
\hline & $\mathrm{H}_{2} \mathrm{O}_{2} 3 \%$ & $55.5 \mathrm{e}$ & $53.5 \mathrm{e}$ & $42.5 \mathrm{~cd}$ & $47.0 \mathrm{de}$ & $49.6 \mathrm{~b}$ \\
\hline & $\mathrm{H}_{2} \mathrm{O}_{2} 6 \%$ & $47.5 \mathrm{de}$ & $36.0 \mathrm{~b}-\mathrm{d}$ & 33.5 a-c & $27.5 \mathrm{a}$ & $36.1 \mathrm{a}$ \\
\hline & $\mathrm{H}_{2} \mathrm{O}_{2} 9 \%$ & $47.0 \mathrm{de}$ & $36.0 \mathrm{a}-\mathrm{c}$ & 36.5 a-c & 33.0 a-c & $38.1 \mathrm{a}$ \\
\hline & $\mathrm{H}_{2} \mathrm{O}_{2} 12 \%$ & $40.5 \mathrm{~cd}$ & $28.0 \mathrm{ab}$ & 35.5 a-c & $33.0 \mathrm{a}-\mathrm{c}$ & $34.3 \mathrm{a}$ \\
\hline & Mean & $54.3 \mathrm{c}$ & $47.5 \mathrm{~b}$ & $45.6 \mathrm{ab}$ & $43.5 \mathrm{a}$ & \\
\hline \multirow{6}{*}{$\begin{array}{l}\text { Fusarium } \\
\text { spp. }\end{array}$} & Water & $80.0 \mathrm{~g}$ & $72.5 \mathrm{fg}$ & $80.0 \mathrm{~g}$ & $81.0 \mathrm{~g}$ & $78.4 \mathrm{~d}$ \\
\hline & $\mathrm{H}_{2} \mathrm{O}_{2} 3 \%$ & 26.0 a-d & 28.0 a-d & $33.5 \mathrm{~d}$ & 21.0 a-d & $27.1 \mathrm{~b}$ \\
\hline & $\mathrm{H}_{2} \mathrm{O}_{2} 6 \%$ & $48.5 \mathrm{e}$ & 60.5 ef & 60.5 ef & $66.0 \mathrm{f}$ & $58.9 \mathrm{c}$ \\
\hline & $\mathrm{H}_{2} \mathrm{O}_{2} 9 \%$ & $29.5 \mathrm{~cd}$ & $18.0 \mathrm{a}-\mathrm{c}$ & $13.0 \mathrm{ab}$ & 19.0 a-d & $19.9 \mathrm{a}$ \\
\hline & $\mathrm{H}_{2} \mathrm{O}_{2} 12 \%$ & 24.5 a-d & $30.0 \mathrm{~cd}$ & $15.5 \mathrm{a}$ & $29.0 \mathrm{~b}-\mathrm{d}$ & $24.8 \mathrm{ab}$ \\
\hline & Mean & $41.7 \mathrm{a}$ & $41.8 \mathrm{a}$ & $40.5 \mathrm{a}$ & $43.2 \mathrm{a}$ & \\
\hline
\end{tabular}

* Means followed by the same letters, for each fungus separately are not significantly different according to the Duncan multiple range test $(\alpha=0.05)$

Further explanations: see Table 1

Table 9. Effects of hydrogen peroxide treatment on the occurrence of fungi on sample II seeds (\%)

\begin{tabular}{|c|c|c|c|c|c|c|}
\hline \multirow{2}{*}{ Fungus } & \multirow{2}{*}{ Treatment } & \multicolumn{4}{|c|}{ Treatment time (min) } & \multirow[b]{2}{*}{ Mean } \\
\hline & & 10 & 20 & 30 & 60 & \\
\hline Alternaria & Water & $71.5 \mathrm{e}$ & $75.0 \mathrm{e}$ & $78.0 \mathrm{e}$ & $77.5 \mathrm{e}$ & $75.5 \mathrm{~d}$ \\
\hline \multirow[t]{5}{*}{ alternata } & $\mathrm{H}_{2} \mathrm{O}_{2} 3 \%$ & $52.0 \mathrm{~d}$ & $46.5 \mathrm{~b}-\mathrm{d}$ & 40.5 a-d & $47.5 \mathrm{~cd}$ & $46.6 \mathrm{c}$ \\
\hline & $\mathrm{H}_{2} \mathrm{O}_{2} 6 \%$ & $43.5 \mathrm{~b}-\mathrm{d}$ & 37.0 a-d & 38.5 a-d & 37.5 a-d & $39.1 \mathrm{ab}$ \\
\hline & $\mathrm{H}_{2} \mathrm{O}_{2} 9 \%$ & $49.0 \mathrm{~cd}$ & $35.0 \mathrm{a}-\mathrm{c}$ & $43.5 \mathrm{~b}-\mathrm{d}$ & $43.0 \mathrm{~b}-\mathrm{d}$ & $42.6 \mathrm{bc}$ \\
\hline & $\mathrm{H}_{2} \mathrm{O}_{2} 12 \%$ & 40.5 a-d & $31.0 \mathrm{ab}$ & 39.5 a-d & $25.5 \mathrm{a}$ & 34.1 a \\
\hline & Mean & $51.3 \mathrm{a}$ & $44.9 \mathrm{a}$ & $48.0 \mathrm{a}$ & $46.2 \mathrm{a}$ & \\
\hline Fusarium & Water & $92.5 \mathrm{~h}$ & $80.5 \mathrm{~g}$ & $78.5 \mathrm{~g}$ & $81.5 \mathrm{~g}$ & $83.3 \mathrm{e}$ \\
\hline \multirow[t]{5}{*}{ spp. } & $\mathrm{H}_{2} \mathrm{O}_{2} 3 \%$ & $22.0 \mathrm{de}$ & $11.5 \mathrm{~cd}$ & $10.0 \mathrm{bc}$ & $11.5 \mathrm{~cd}$ & $13.8 \mathrm{~b}$ \\
\hline & $\mathrm{H}_{2} \mathrm{O}_{2} 6 \%$ & $25.0 \mathrm{e}$ & $25.5 \mathrm{e}$ & $48.5 \mathrm{f}$ & $49.5 \mathrm{f}$ & $37.1 \mathrm{~d}$ \\
\hline & $\mathrm{H}_{2} \mathrm{O}_{2} 9 \%$ & $21.5 \mathrm{de}$ & $21.0 \mathrm{de}$ & $25.5 \mathrm{e}$ & $15.5 \mathrm{c}-\mathrm{e}$ & $20.9 \mathrm{c}$ \\
\hline & $\mathrm{H}_{2} \mathrm{O}_{2} 12 \%$ & $5.0 \mathrm{ab}$ & $3.5 \mathrm{a}$ & $1.5 \mathrm{a}$ & $1.5 \mathrm{a}$ & $2.9 \mathrm{a}$ \\
\hline & Mean & $33.2 \mathrm{~b}$ & $28.4 \mathrm{a}$ & $32.8 \mathrm{ab}$ & $31.9 \mathrm{ab}$ & \\
\hline
\end{tabular}

* Means followed by the same letters, for each fungus separately, are not significantly different according to the Duncan multiple range test $(\alpha=0.05)$

Further explanations: see Table 1

\section{DISCUSSION}

Two samples of zinnia seeds, varying significantly in germination capacity and incidence of fungi, in particular pathogenic A. zinniae, were used in the experiment. Hydrogen peroxide treatment improved the germination capacity of sample I, characterised by a high infestation of this pathogen, but negatively affected this parameter in the seeds of sample II free from $A$. zinniae and with a high initial germination. In previous experiments, the low percentage of normal seedlings in zinnia seed samples with relatively high germination 
Table 10. Effects of fungicide and hydrogen peroxide treatment on germination of zinnia seeds

\begin{tabular}{|c|c|c|c|c|c|}
\hline \multirow{2}{*}{ Sample } & \multirow{2}{*}{ Treatment } & \multirow{2}{*}{$\begin{array}{l}\text { Total germination } \\
\left(\mathrm{G}_{\max }\right)(\%)\end{array}$} & \multirow{2}{*}{$\begin{array}{c}\text { Germination capacity } \\
(\%)\end{array}$} & \multicolumn{2}{|c|}{ Abnormal seedlings (\%) } \\
\hline & & & & Deformed & Diseased \\
\hline \multirow[t]{4}{*}{ I } & Control* & $83.8 \mathrm{~b}^{* *}$ & $39.5 \mathrm{a}$ & $0.5 \mathrm{a}$ & $41.3 \mathrm{~b}$ \\
\hline & Fungicide & $86.5 \mathrm{~b}$ & $49.3 \mathrm{~b}$ & $2.5 \mathrm{a}$ & $32.8 \mathrm{ab}$ \\
\hline & $\mathrm{H}_{2} \mathrm{O}_{2} 3 \% 10 \mathrm{~min}$ & $84.0 \mathrm{~b}$ & $47.8 \mathrm{ab}$ & $1.3 \mathrm{a}$ & 30.8 a \\
\hline & $\mathrm{H}_{2} \mathrm{O}_{2} 3 \% 20 \mathrm{~min}$ & $84.8 \mathrm{~b}$ & $50.3 \mathrm{~b}$ & $1.3 \mathrm{a}$ & $30.0 \mathrm{a}$ \\
\hline \multirow[t]{4}{*}{ II } & Control & $94.5 \mathrm{a}$ & $87.0 \mathrm{~b}$ & $0.8 \mathrm{a}$ & $3.3 \mathrm{a}$ \\
\hline & Fungicide & $94.3 \mathrm{a}$ & $74.3 \mathrm{a}$ & $5.3 \mathrm{~b}$ & $10.3 \mathrm{ab}$ \\
\hline & $\mathrm{H}_{2} \mathrm{O}_{2} 3 \% 10 \mathrm{~min}$ & $93.5 \mathrm{a}$ & $75.0 \mathrm{a}$ & $0.3 \mathrm{a}$ & $14.8 \mathrm{~b}$ \\
\hline & $\mathrm{H}_{2} \mathrm{O}_{2} 3 \% 20 \mathrm{~min}$ & $92.0 \mathrm{a}$ & $75.5 \mathrm{a}$ & $4.3 \mathrm{~b}$ & $15.0 \mathrm{~b}$ \\
\hline
\end{tabular}

*Control - untreated seeds; Fungicide - seeds treated with Sarfunt T 65 DS at a dose $5 \mathrm{~g}$ per $1 \mathrm{~kg}$ of seeds; $\mathrm{H}_{2} \mathrm{O}_{2} 3 \% 10$ min - seeds soaked in 3\% hydrogen peroxide solution for $10 \mathrm{~min} ; \mathrm{H}_{2} \mathrm{O}_{2} 3 \% 20 \mathrm{~min}$ - seeds soaked in 3\% hydrogen peroxide solution for $20 \mathrm{~min}$ **Means in columns followed by the same letters, for each sample separately, are not significantly different according to the Duncan multiple range test $(\alpha=0.05)$

Table 11. Effects of fungicide and hydrogen peroxide treatment on vigour of zinnia seeds

\begin{tabular}{llcc}
\hline Sample & Treatment & T1* (days) & MGT (days) \\
\hline I & Control & $0.63 \mathrm{~b}$ & $1.48 \mathrm{~b}$ \\
& Fungicide & $0.93 \mathrm{c}$ & $2.13 \mathrm{c}$ \\
& $\mathrm{H}_{2} \mathrm{O}_{2} 3 \% 10 \mathrm{~min}$ & $0.44 \mathrm{a}$ & $1.31 \mathrm{ab}$ \\
& $\mathrm{H}_{2} \mathrm{O}_{2} 320 \mathrm{~min}$ & $0.77 \mathrm{~b}$ & $1.47 \mathrm{~b}$ \\
\hline $\mathrm{II}$ & Control & $0.78 \mathrm{bc}$ & $1.44 \mathrm{ab}$ \\
\hline & Fungicide & $0.84 \mathrm{~d}$ & $2.07 \mathrm{c}$ \\
& $\mathrm{H}_{2} \mathrm{O}_{2} 310 \mathrm{~min}$ & $0.34 \mathrm{a}$ & $1.59 \mathrm{~b}$ \\
& $\mathrm{H}_{2} \mathrm{O}_{2} 320 \mathrm{~min}$ & $0.74 \mathrm{c}$ & $1.57 \mathrm{ab}$ \\
\hline
\end{tabular}

*T1 - time needed to $1 \%$ of total germinating seeds; MGT - mean germination time Further explanations: see Table 10

percentages was usually associated with significant fungal seed infestation, resulting in the occurrence of a large number of abnormal diseased seedlings during germination tests (Szopińska and Tylkowska 2009, Szopińska and Wojtaszek 2011). There are many reports that the promotion of seed germination by hydrogen peroxide treatment may be the result of the oxidation of germination inhibitors present in the seed coat or/and in the pericarp (Duval and NeSmith 2000, Ogawa and Iwabuchi 2001, Conner 2008, Klein et al. 2008, Huarte and Garcia 2009). Klein et al. (2008) found that faster germination of eastern gamagrass seeds exposed to hydrogen peroxide may result

Table 12. Effects of fungicide and hydrogen peroxide treatment on health of zinnia seeds

\begin{tabular}{|c|c|c|c|c|c|}
\hline \multirow{2}{*}{ Sample } & \multirow{2}{*}{ Treatment } & \multirow{2}{*}{$\begin{array}{l}\text { Seeds free from fungi } \\
\qquad(\%)\end{array}$} & \multicolumn{3}{|c|}{ Seed infestation with fungi (\%) } \\
\hline & & & Alternaria alternata & Alternaria zinniae & Fusarium spp. \\
\hline \multirow[t]{4}{*}{ I } & Control & $0 \mathrm{a}$ & $76.5 \mathrm{c}$ & $76.0 \mathrm{c}$ & $52.0 \mathrm{c}$ \\
\hline & Fungicide & $18.5 \mathrm{~cd}$ & $41.5 \mathrm{ab}$ & $51.0 \mathrm{~b}$ & $2.5 \mathrm{a}$ \\
\hline & $\mathrm{H}_{2} \mathrm{O}_{2} 3 \% 10 \mathrm{~min}$ & $10.5 \mathrm{~b}$ & $48.0 \mathrm{~b}$ & $55.5 \mathrm{~b}$ & $26.0 \mathrm{~b}$ \\
\hline & $\mathrm{H}_{2} \mathrm{O}_{2} 3 \% 20 \mathrm{~min}$ & $16.5 \mathrm{bc}$ & $36.0 \mathrm{a}$ & $53.5 \mathrm{~b}$ & $28.0 \mathrm{~b}$ \\
\hline \multirow[t]{4}{*}{ II } & Control & $5.0 \mathrm{a}$ & $78.0 \mathrm{~d}$ & $-*$ & $38.5 \mathrm{c}$ \\
\hline & Fungicide & $71.0 \mathrm{c}$ & $25.0 \mathrm{a}$ & - & $6.0 \mathrm{a}$ \\
\hline & $\mathrm{H}_{2} \mathrm{O}_{2} 3 \% 10 \mathrm{~min}$ & $41.0 \mathrm{~b}$ & $52.0 \mathrm{c}$ & - & $22.0 \mathrm{~b}$ \\
\hline & $\mathrm{H}_{2} \mathrm{O}_{2} 3 \% 20 \mathrm{~min}$ & $50.5 \mathrm{~b}$ & $46.5 \mathrm{bc}$ & - & $11.5 \mathrm{a}$ \\
\hline
\end{tabular}

*In sample II Alternaria zinniae was not detected on the seeds Further explanations: see Table 10 
from breaking down the inhibitors present in the pericarp, although there may also be a direct physical scarifying effect of this compound. Ogawa and Iwabuchi (2001) discussed the possibility that endogenously generated $\mathrm{H}_{2} \mathrm{O}_{2}$ functions as a promoter of zinnia seed germination by oxidizing germination inhibitors. Narimanov (2000) observed that short seed treatment in $\mathrm{H}_{2} \mathrm{O}_{2}$ solution promoted the early appearance of sprouts, and accelerated the development of barley, maize, haricot, melon, vegetable marrow, garden radish and carrot. The author suggests that the seeds exit from dormancy as a result of the oxidation reaction catalysed by NADPH oxidase. In both samples in the present experiment seedlings appeared very quickly, within the first few days of the germination test, regardless of treatment. Moreover, an acceleration of seed germination after treatment with $\mathrm{H}_{2} \mathrm{O}_{2}$ was caused by the conditioning effect, obtained by soaking seeds in distilled water itself, rather than the actual activity of hydrogen peroxide. On the other hand, a prolonged treatment of the seeds with $\mathrm{H}_{2} \mathrm{O}_{2}$ at the highest concentrations resulted in a significant reduction in seed germination and vigour, especially in sample II. This deleterious effect was also observed by Sasaki et al. (2005). The authors found that the imbibition of rice seeds in 5, 50 and 100 $\mathrm{mM} \mathrm{H}_{2} \mathrm{O}_{2}$ solutions for $24 \mathrm{~h}$ significantly increased the shoot fresh weight of the seedlings, while treatment with higher concentrations (500 and 1000 $\mathrm{mM}$ of $\mathrm{H}_{2} \mathrm{O}_{2}$ ) reduced this value. Nevertheless, it can be assumed that the improvement of sample I seed germination after treatment was a result of the antifungal activity of hydrogen peroxide rather than the effect of the oxidation of germination inhibitors. The antimicrobial properties of hydrogen peroxide were reported previously by some researchers (Peng and Kuc 1992, Smilanick et al. 1994, Geetha and Shetty 2002). Geetha and Shetty (2002) found that treatment of pearl millet (Pennisetum glaucum) seeds with $1 \mathrm{mM} \mathrm{H}_{2} \mathrm{O}_{2}$, effectively suppressed the development of downy mildew caused by Sclerospora graminicola Sacc. Schroet., which resulted in an increase of the vegetative and reproductive growth parameters and enhanced grain yield. Smilanick et al. (1994) observed that a 6 min treatment of barley and wheat seeds at $3.5 \% \mathrm{H}_{2} \mathrm{O}_{2}$ completely inhibited the growth of Tilletia controversa and T. tritici spores at $45^{\circ} \mathrm{C}$ and $50^{\circ} \mathrm{C}$. Peng and Kuc (1992) found that hydrogen peroxide at a $2.6110^{-5} \mathrm{M}$ concentration inhibited the germination of Cladosporium cucumerinum, Colletotrichum lagenarium and Peronospora tabacina spores in vitro. Moreover, peroxidasegenerated $\mathrm{H}_{2} \mathrm{O}_{2}$ inhibited the development of blue mould ( $P$. tabacina) on tobacco leaf disks. The authors suggest that $\mathrm{H}_{2} \mathrm{O}_{2}$ may function as an antifungal factor in disease resistance by the direct inhibition of pathogen development, and/ or by the generation of other reactive free radicals that are antimicrobial. In the present experiment, treatment with $\mathrm{H}_{2} \mathrm{O}_{2}$ resulted in a significant increase of the percentage of seeds free from fungi in both samples. This phenomenon was associated mostly with a decrease of seed infestation with $A$. alternata, A. zinniae and Fusarium spp. in sample I and A. alternata and Fusarium spp. and in sample II. An increase in the number of seeds free from fungi after the treatment of China aster and onion seeds with $3 \% \mathrm{H}_{2} \mathrm{O}_{2}$ was also observed by Rosada (2012) and Słupinska (2012). Furthermore, Rosada (2012) reported that hydrogen peroxide treatment significantly reduced infestation of China aster seeds with A. alternata, A. strictum, Cladosporium spp. and Fusarium spp., whereas Słupinska (2012) found that soaking seeds of onion in $3 \% \mathrm{H}_{2} \mathrm{O}_{2}$ solution limited the occurrence of A. alternata, Aureobasidium sp., Botrytis aclada, Cladosporium spp., Rhizopus sp. and Stemphylium spp. Hydrogen peroxide treatment was also effective against pathogenic Fusarium species occurring on seeds of conifer plants (Neumann et al. 1997).

The reduction of sample II seed germination and vigour after applied treatments, and the improvement of these parameters in sample I, characterised initially with poor germination and very high level of infection with $A$. zinniae, suggest that hydrogen peroxide treatment should be preceded by seed health evaluation. Otherwise, it may lead to a deterioration of seed quality, especially if higher concentrations of hydrogen peroxide are used for seed treatment.

\section{CONCLUSION}

1. The treatment of zinnia seeds with hydrogen peroxide, regardless of time and concentration of $\mathrm{H}_{2} \mathrm{O}_{2}$, significantly reduced seed infestation with fungi in both samples. However, the lowest percentage of seeds infected with $A$. zinniae was observed if the seeds were soaked in $6 \%$ and $12 \% \mathrm{H}_{2} \mathrm{O}_{2}$ solutions for 60 and $20 \mathrm{~min}$, respectively.

2. The treatment of zinnia seeds in a $3 \%$ solution of $\mathrm{H}_{2} \mathrm{O}_{2}$ for 20 min positively influenced seed germination, especially in sample I, infested 
with A. zinniae and characterised initially by a low germination capacity. However, soaking seeds of both samples in $3 \% \mathrm{H}_{2} \mathrm{O}_{2}$ solution for 10 min significantly improved their vigour, expressed by the $T_{1}$ parameter.

3. It can be assumed that the treatment of zinnia seeds with hydrogen peroxide can effectively improve germination mostly in samples strongly infected with pathogens, which means that it should be preceded by seed health evaluation.

\section{REFERENCES}

Chien C.T., Lin T.P., 1994. Mechanism of hydrogen peroxide in improving the germination of Cinnamonum camphora seed. Seed Sci. Technol. 22: 231-236.

Conner P.J., 2008. Effects of stratification, germination temperature and pre-treatment with gibberellic acid and hydrogen peroxide on germination of 'Fry' muscadine (Vitis rotundifolia) seed. HortSci. 43(3): 853-856.

Dimock A.W., Osborn J.H., 1943. An Alternaria disease of zinnia. Phytopathology 33: 372-381.

Duval J.R., NeSmith D.S., 2000. Treatment with hydrogen peroxide and seedcoat removal or clipping improve germination of 'Genesis' triploid watermelon. HortScience 35: 85-86.

Geetha H.M., Shetty H.S. 2002. Induction of resistance in pearl millet against downy mildew disease caused by Sclerospora graminicola using benzothiadiazole, calcium chloride and hydrogen peroxide a comparative evaluation. Crop Prot. 21(8): 601-610.

Groot S.P.C., VAn der Wolf J.M., Jalink H., Langerak C.J., VAN DEN BulK R.W., 2004. Challanges for the production of high quality organic seeds. Seed Testing International 127: 12-15.

Huarte R., Garcia M.D., 2009. Tripsacum dactyloides (L.) L. (Poaceae) caryopsis dormancy and germination responses to scarification, hydrogen peroxide and phytohromes. Seed Sci. Technol. 37: 544-553.

ISTA, 2012. International Rules for Seed Testing. International Seed Testing Association, Bassersdorf, Switzerland.

Jalink H., VAn der Schoor R., 1999. Seed Calculator 2.1. License number: 100200122. Plant Research International, Wageningen, the Netherlands.

Jaskani M.J., Kwon S.W., Kim D.H., AbBas H., 2006. Seed treatments and orientation affects germination and seedling emergence in tetraploid watermelon. Pakistan J. Bot. 38(1): 89-98.

Klein J.D., Wood L.A., Geneve R.L., 2008. Hydrogen peroxide and color sorting improves germination and vigor of eastern gamagrass (Tripsacum dactyloides) seeds. Acta Hortic. 782: 93-97.
Łacicowa B., Filipowicz A., Wagner A., 1979. The fungi pathogenic for Zinnia elegans L. Acta Mycol. 15(1): 11-20 (In Polish).

Łacicowa B., Kiecana I., Pięta D., 1991. Mycoflora of ornamental plants seeds. I. Mycoflora of zinnia (Zinnia elegans L.) and sweet pea seeds (Lathyrus odoratus L.). Rośliny Ozdobne, Prace ISiK, Seria B 16: 109-116 (In Polish)

LeHrer R.I., 1969. Antifungal effects of peroxidase systems. J. Bacteriol. 99(2): 361-365.

Machado J.C., Langerak C.J., Jaccoud-Filho D.S., 2002. Seed-borne Fungi: A Contribution to Routine Seed Health Analysis. International Seed Testing Association. Bassersdorf, Switzerland.

Mathur S.B., Kongsdal O., 2003. Common laboratory seed health testing methods for detecting fungi. International Seed Testing Association, Bassersdorf, Switzerland.

Naredo M.E.B., Juliano A.B., Lu B.R., Guzman de F., JACKSON M., 1998. Responses to seed dormancybreaking treatments in rice species (Oryza L.). Seed Sci. Technol. 26: 675-689.

Narimanov A.A., 2000. Presowing treatment of seeds with hydrogen peroxide promotes germination and development of plants. Biológia (Bratislava) 55(4): 425-428.

Neumann M., Trotter D., Kolotelo D., 1997. Seed sanitation method to reduce seedborne Fusarium levels on conifer seed. Surrey, BC: British Columbia Ministry of Forests, Seed and Seedling Extension Topics 10(1\&2): 18-23.

Ogawa K., Iwabuchi M., 2001. A mechanism for promoting the germination of Zinnia elegans seeds by hydrogen peroxide. Plant Cell Physiol. 42(3): 286291.

Peng M., Kuc J., 1992. Peroxidase-generated hydrogen peroxide as a source of antifungal activity in vitro and on tobacco leaf disks. Phytopathology 82: 696699.

RiCHARDSON M.J. 1990. An annotated list of seed-borne diseases. International Seed Testing Association. Zürich. Switzerland.

RosadA D., 2012. Effects of hydrogen peroxide and organic acids on germination, vigour and health of China aster (Callistephus chinensis Nees.) seeds. MSc thesis, Poznań University of Life Sciences, Poland (In Polish with English summary).

SAPERS G.M., Sites J.E., 2003. Efficacy of 1\% hydrogen peroxide wash in decontaminating apples and cantaloupe melons. J. Food Sci. 68(5): 1793-1797.

Sasaki K., Kishtami S., Fumitaka A., Sato T., 2005. Promotion of seedling growth of seeds of rice (Oryza sativa L. cv. Hitomebore) by treatment with $\mathrm{H}_{2} \mathrm{O}_{2}$ before sowing. Plant Prod. Sci. 8(5): 509-514.

Smilanick J.L., Goates B.J., Denis-Arrue R., Simmons G.F., Peterson G.L., Henson D.J. et AL., 1994. Germinability of Tilletia spp. after hydrogen peroxide treatment. Plant Dis. 78: 861-865. 
SŁUPINSKA E., 2012. Effects of hydrogen peroxide and organic acids on germination, vigour and health of onion (Allium cepa L.) seeds. MSc thesis, Poznań University of Life Sciences, Poland (In Polish with English summary).

Szopińska D., Tylkowska K., Deng CH.J., Gao Y., 2012. Comparison of modified blotter and agar incubation methods for detecting fungi in Zinnia elegans seeds. Seed Sci. Technol. 40: 32-42.

Szopińska D., TYlkowsKa S., 2009. Effect of osmopriming on germination and location of fungi in Zinnia elegans Jacq. seeds. Phytopathologia 54: 33-44.

Szopińska D., WoJTASzeK A., 2011. Effect of hydropriming on germination and location of fungi in Zinnia elegans Jacq. seeds. Nauka Przyr. Technol. $5,6,117$.

Thомаs E.L., 1979. Myeloperoxidase, hydrogen peroxide, chloride antimicrobial system: Nitrogenchlorine derivatives of bacterial components in bacterial action against Escherichia coli. Infect. Immun. 23(2): 522-531.

Wahid A., Sehar S., Perveen M., Gelani S., Basra S.M.A., FAROoQ M., 2008. Seed pre-treatment with hydrogen peroxide improves heat tolerance in maize at germination and seedling growth stages. Seed Sci. Technol. 36(3): 633-645.

Wu W.S., YANG Y.H., 1992. Alternaria blight, a seedtransmitted disease of zinnia in Taiwan. Plant Pathol. Bull. 1: 115-123.

\section{WPEYW TRAKTOWANIA NADTLENKIEM WODORU NA KIEŁKOWANIE, WIGOR I ZDROWOTNOŚĆ NASION ZINNIA ELEGANS}

Streszczenie: Znaczące zasiedlenie nasion cynii (Zinnia elegans Jacq.) przez grzyby, zwłaszcza przez patogena Alternaria zinniae, często skutkuje pogorszeniem zdolności kiełkowania nasion. W doświadczeniu badano wpływ nadtlenku wodoru $\left(\mathrm{H}_{2} \mathrm{O}_{2}\right)$ na kiełkowanie, wigor i zdrowotność nasion cynii. Testowano dwie próby nasion: próbę I charakteryzującą się niską zdolnością kiełkowania (39.5\%) i wysokim poziomem porażenia nasion przez A. zinniae $(76 \%)$ oraz próbę II - odznaczającą się wysoką zdolnością kiełkowania (87\%) i wolną od tego patogena. Nasiona traktowano poprzez moczenie w 3\%, $6 \%, 9 \%$ i $12 \%$ roztworach $\mathrm{H}_{2} \mathrm{O}_{2}$ przez $10,20,30$ i 60 min. Kontrolę stanowiły początkowo nasiona moczone w wodzie destylowanej przez 10, 20, 30 i $60 \mathrm{~min}$, a następnie wyniki wybranych wariantów traktowania porównywano $\mathrm{z}$ wynikami uzyskanymi dla nasion nietraktowanych i nasion traktowanych fungicydem, zawierającym $20 \%$ karbendazymu i $45 \%$ tiuramu. $\mathrm{Na}$ nasionach najczęściej identyfikowano grzyby rodzajów Alternaria i Fusarium. Stężenie nadtlenku wodoru w większym stopniu wpływało na jakość nasion cynii niż czas traktowania. Niemniej, zastosowane traktowanie, bez względu na czas jego trwania i stężenie $\mathrm{H}_{2} \mathrm{O}_{2}$, korzystnie wpływało na zdrowotność nasion obu prób, znacząco ograniczając ich zasiedlenie przez grzyby. Ponadto, najniższy poziom infekcji przez A. zinniae obserwowano po traktowaniu nasion $6 \%$ i $9 \%$ roztworem $\mathrm{H}_{2} \mathrm{O}_{2}$ przez odpowiednio 60 i 20 min. Nadtlenek wodoru w wyższych stężeniach ( $9 \%$ i $12 \%$ ) negatywnie wpływał na kiełkowanie i wigor nasion, zwłaszcza u próby II. Można wnioskować, że traktowanie nasion cynii nadtlenkiem wodoru skutecznie poprawia ich kiełkowanie głównie u prób silnie porażonych przez patogeny, co oznacza, że powinno być ono poprzedzone oceną zdrowotności nasion.

Received June 27, 2013; accepted December 19, 2013 